\title{
Regulation of Self-renewal and Differentiation in Adult Stem Cell Lineages: Lessons from the Drosophila Male Germ Line
}

\author{
E.L. DAVIES AND M.T. FulleR \\ Departments of Developmental Biology and Genetics, Stanford University \\ School of Medicine, Stanford, California 94305-5329
}

\begin{abstract}
The ability to identify stem cells and trace their descendants in vivo has yielded insights into how self-renewal, proliferation, and differentiation are regulated in adult stem cell lineages. Analysis of male germ-line stem cells in Drosophila has revealed the importance of local signals from the microenvironment, the stem cell niche, in controlling stem cell behavior. Germ-line stem cells physically attach to the niche via localized adherens junctions that provide a polarity cue for orientation of centrosomes in interphase and the spindle in mitosis. As a result, stem cells divide asymmetrically: One daughter inherits attachment to the niche and remains within its embrace, whereas the other is displaced away and initiates differentiation. Strikingly, much as leukemia inhibitory factor (LIF) and transforming growth factor- $\beta$ (TGF- $\beta$ ) signaling maintain mouse embryonic stem (ES) cells, maintenance of stem cell state in the Drosophila male germ line is regulated by cytokine-like signals from hub cells that activate the transcription factor STAT (signal transducer and activator of transcription) and TGF- $\beta$ class signals from surrounding support cells that repress expression of a key differentiation factor. Surprisingly, transit-amplifying cells can revert to the stem cell state if they reoccupy the niche. Upon cessation of mitosis and the switch to terminal differentiation, germ cells express cell-type- and stage-specific transcription machinery components that drive expression of terminal differentiation genes, in part by removing Polycomb transcriptional silencing machinery.
\end{abstract}

Regulated activity of adult stem cells is essential for tissue homeostasis. Adult stem cell lineages are responsible for the long-term maintenance and repair of tissues containing highly specialized, short-lived cell types, including blood, sperm, skin, and intestinal epithelium. Recent studies suggest that differentiated cells in other tissues, including breast, lung, skeletal muscle, and prostate, are also produced from adult stem cells in response to physiological changes or damage. Hallmarks of adult stem cells are a committed but relatively undifferentiated state, long-term ability to proliferate, and ability to produce both new stem cells (self-renewal) and differentiating progeny (Fig. 1). Understanding the mechanisms that regulate adult stem cell self-renewal, as well as those that regulate the proliferation and differentiation of their progeny, will be key for harnessing the potential of adult stem cells for regenerative medicine and may also suggest new strategies for the war on cancer. Many common cancers arise in adult stem cell lineages, and there is increasing evidence that defects in the mechanisms that regulate self-renewal, proliferation, and differentiation in adult stem cell lineages can contribute to tumorigenesis (Clarke and Fuller 2006).

\section{KEY REGULATORY POINTS IN ADULT STEM CELL LINEAGES}

Tissue homeostasis in adult stem cell lineages requires the proper execution and coordination of several key switches in cell state (Fig. 1A). When adult stem cells

A Stem cell

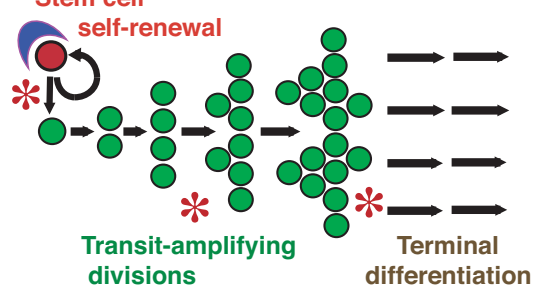

B

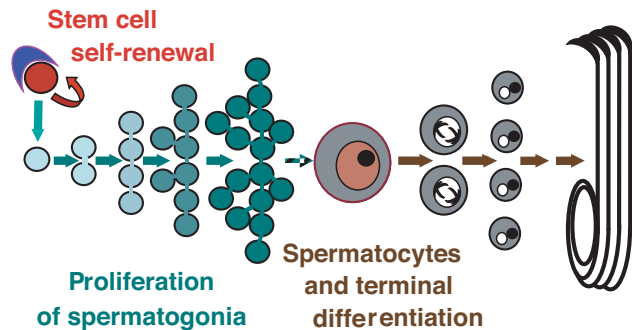

Figure 1. Key decisions in adult stem cell lineages. (A) An archetypical adult stem cell lineage. A stem cell (red) in the niche (blue crescent) divides to both self-renew and produce a daughter that initiates differentiation (green). After a limited series of transit-amplifying mitotic divisions (green), the cells cease proliferation and turn on the terminal differentiation program (bank of arrows). Asterisks mark key regulatory points: (Left to right) Self-renewal versus onset of differentiation; limiting the number of transient-amplifying divisions; switch to terminal differentiation. (B) Male germ-line stem cell lineage in Drosophila. A stem cell (red) in the niche at the tip of the testis divides asymmetrically, producing a new stem cell and a gonialblast, which initiates four rounds of synchronous spermatogonial mitotic divisions with incomplete cytokinesis (green arrows). The resulting 16 interconnected germ cells undergo premeiotic DNA replication in synchrony and turn on the spermatocyte program of cell growth, meiotic prophase, and transcription of terminal differentiation genes (large cell with brown nucleus: All 16 cells become spermatocytes, but only one is shown for simplicity). After the two meiotic divisions, the 64 haploid spermatids undergo complete remodeling to generate mature sperm. 
divide, the daughter cells must first choose between maintenance of stem cell identity or initiation of differentiation. Specialized microenvironments, known as stem cell niches, regulate the outcome of this critical cell-fate decision in vivo, ensuring that populations of stem cells and differentiating progeny are properly maintained (Watt and Hogan 2000; Spradling et al. 2001; Yamashita et al. 2005; Fuller and Spradling 2007). In several well-studied adult stem cell model systems, stem cell self-renewal relies on close-range signals from a support cell niche; failure to maintain an intimate association with the niche causes cells to adopt differentiated fates. Discrete anatomical niches provide a strategy for regulating stem cell number and may defend against cancer by preventing undifferentiated precursors from self-renewing outside of the niche. In most systems, however, the difficulty in identifying stem cells in situ has precluded investigations of the relationship between stem cells and their natural environment.

In many adult stem cell lineages, stem cell daughters that initiate differentiation execute a series of transit-amplifying divisions before terminal differentiation, thereby allowing a large number of differentiated cells to be produced from a single stem cell division (Fig. 1A). Mechanisms that limit the number of transit-amplifying cell divisions comprise another key regulatory point: They ensure that mutations arising in precursor cells are not maintained in the pool of long-term proliferating cells. Third, the switch from proliferation to terminal differentiation entails dramatic changes in the cell cycle and gene expression programs, culminating in expression of large numbers of terminal differentiation genes that were either silenced or expressed at only low levels in the precursor cells. Additionally, many adult stem cell lineages are multipotent, and precursor cells must choose to differentiate along a single, context-appropriate developmental pathway.

Investigation of the male and female germ-line stem cell (GSC) lineages in Drosophila has provided insight into the types of mechanisms that regulate these critical cell-fate switches in vivo and paradigms for researchers working in mammalian stem cell systems. In Drosophila, GSC self-renewal depends on short-range signals from a support cell niche (Xie and Spradling 1998; Kiger et al. 2001; Tulina and Matunis 2001). Other key features include physical attachment to the niche, orientation of stem cell divisions with respect to the niche (Xie and Spradling 2000; Yamashita et al. 2003, 2007), and the ability of transit-amplifying cells that reoccupy the niche to revert to stem cell function (Brawley and Matunis 2004; Kai and Spradling 2004; Cheng et al. 2008). For a review of research on the female GSC niche, see Spradling et al. (this volume). Here, we discuss the mechanisms that regulate self renewal and differentiation in the Drosophila male GSC niche. Comparison of these two systems has revealed key conserved features and also interesting differences.

\section{SPERMATOGENESIS AS A MODEL ADULT STEM CELL SYSTEM}

Male gametes are produced in a unipotent adult stem cell lineage (Fig. 1B). In Drosophila, spermatogenesis ini- tiates with the asymmetric division of a male GSC. One daughter maintains stem cell identity and the other initiates differentiation as a gonialblast (Gb), the founder of a clone of transit-amplifying spermatogonia (Figs. 1A and 2C). Transit-amplifying spermatogonia can be clearly distinguished from GSCs on the basis of cell behavior, structure, and gene expression. GSCs divide asynchronously, execute complete cytokinesis, and contain a ball-shaped spectrin-rich intracellular membranous organelle called the spectrosome (Fig. 2C). In contrast, transit-amplifying spermatogonia descended from a $\mathrm{Gb}$ divide synchronously with incomplete cytokinesis, producing a cyst of interconnected germ cells joined by cytoplasmic bridges. As spermatogonial cysts grow with each round of mitosis, the ball-shaped spectrosome elongates and threads through the ring canals that connect germ cells within a cyst, creating a branched organelle called the fusome (Fig. 2C). After undergoing a genetically predetermined number of transitamplifying divisions (four in Drosophila melanogaster), the resulting 16 germ cells together exit the mitotic program and commit to terminal differentiation. The cells synchronously execute premeiotic DNA synthesis, adopt primary spermatocyte fate, grow dramatically in volume, and turn on an extremely active cell-type-specific transcription program in preparation for terminal differentiation (Fig. 1A). Many genes are transcribed for the first time in development in primary spermatocytes, and many widely expressed genes are transcribed from alternate spermatocyte-specific promoters (for review, see Fuller 1993; Hecht 1993). The 16 spermatocytes then together undergo the two meiotic divisions, and the resulting 64 haploid spermatids execute a dramatic remodeling program to generate mature sperm.

\section{THE TESTIS STEM CELL NICHE MAINTAINS TWO ADULT STEM CELL POPULATIONS}

Drosophila male GSCs reside in a niche that instructs their self-renewal. Approximately eight to ten GSCs lie in a rosette around a cluster of somatic support cells called the hub at the apical tip of each testis (Fig. 2). The hub supports stem cell self-renewal by serving as the exclusive source of the signaling ligand Unpaired ( $U p d$ ) (Fig. 2A). In a cytokine-like signaling pathway, Upd produced by hub cells locally activates the Janus kinase-signal transducer and activator of transcription (JAK-STAT) pathway in neighboring cells. Action of STAT is required cell autonomously in male GSCs for stem cell maintenance: Male GSCs made homozygous mutant for a null allele of stat fail to maintain stem cell identity and instead differentiate (Kiger et al. 2001; Tulina and Matunis 2001). Agedependent decrease in Upd expression in the hub may contribute to GSC loss with advanced age (Boyle et al. 2007).

The apical hub serves as a niche for two adult stem cell populations: male GSCs and somatic cyst stem cells (CySCs) (Figs. 2C and 3), allowing spatially coordinated production of progeny cells that interact and codifferentiate. The cell bodies of CySCs sit distal to the GSCs, and they maintain direct contact with the hub via narrow cytoplasmic processes that intercalate between the GSCs. 

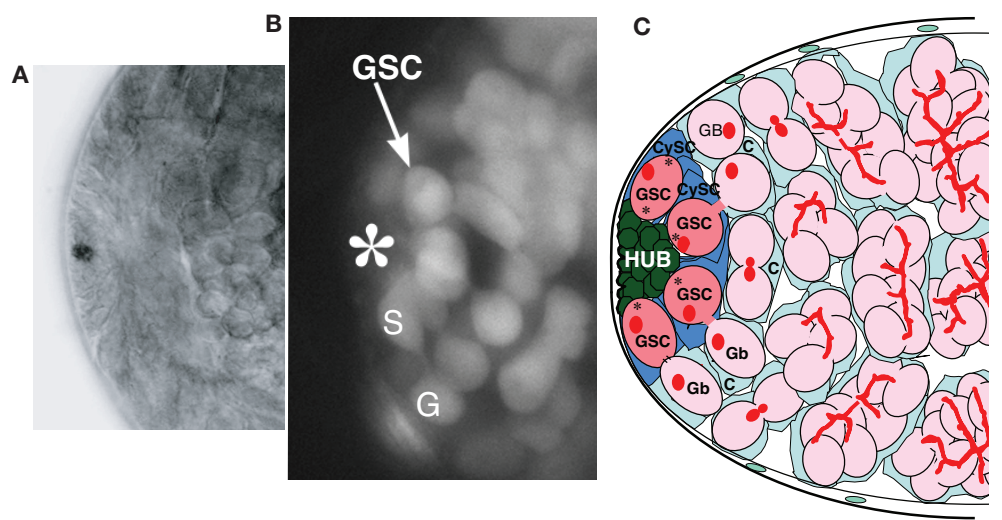

Figure 2. Stem cells in the niche at the apical tip of the Drosophila testis. (A) Hub cells at the apical tip of the testis express the signaling ligand $U p d$. In situ hybridization shows local expression of $U p d$ mRNA. (B) Male germ cells at the apical tip of the testis viewed live by germ-line-specific expression of UAS-GFP under control of nos-GAL4. Asterisk indicates the apical hub composed of somatic cells. (S) GSC next to the hub; (G) gonialblast displaced away from the hub. Note the transient cytoplasmic bridge connecting the stem cell and the gonialblast, indicating that the two arose recently from division of a stem cell. (C) Diagram of the testis stem cell niche and germinal proliferation center. GSCs (dark pink) attach to hub cells (green) and are flanked by somatic cyst stem cells (CySC) (dark blue). Gonialblasts $(\mathrm{Gb})$ produced by GSCs are displaced away from the hub and enveloped by two somatic cyst cells (C) (light blue) produced by the CySCs. Each gonialblast finds a mitotic clone of spermatogonia (light pink) that divide in synchrony, forming cysts of 2, 4, 8, and 16 interconnected germ cells. (Red balls) Spectrosomes; (red lines) fusomes. The germ cell cysts execute premeiotic S and become spermatocytes (not shown) ( $A$, Reprinted, with permission, from Kiger et al. 2001 [CAAAS]; $C$, reprinted, with permission, from Fuller and Spradling 2007 [CAAAS].)

CySCs self-renew and give rise to daughters that differentiate into somatic cyst cells (Gönczy and DiNardo 1996). Cyst cells occasionally undergo one round of transitamplifying division, but they usually adopt a postmitotic fate and initiate differentiation immediately after the CySC division (Voog et al. 2008). Two cyst cells associate with and envelop each gonialblast, forming a discrete packet, or cyst (Fig. 3), that remains intact throughout the rest of spermatogenesis. The mechanisms that specify the ratio of one $\mathrm{Gb}$ to two cyst cells are not yet understood. The cyst cells codifferentiate with the germ cells that they enclose (Gönczy et al. 1992), and continued association and communication between the germ line and the enveloping somatic cyst cells is essential for proper differentiation of both cell types. CySCs are bipotential: CySCs or their daughters occasionally contribute to the hub (Voog et al. 2008), suggesting a novel mechanism for maintaining the hub. This relationship may reflect the common segmental origin of hub and CySCs in the embryonic gonad (Le Bras and Van Doren 2006). The choice between cyst cell versus hub cell identity may be influenced by the presence of germ cells, because the hub expands and changes morphology in agametic testes (Gönczy and DiNardo 1996; Voog et al. 2008). The picture emerging is that stem cells and their niches are mutually interdependent self-sustaining systems.

The same cytokine-like signal from the hub that maintains GSCs also maintains the somatic CySCs (Fig. 3). Like GSCs, the CySCs overproliferate when $U p d$ is ectopically expressed in early germ cells (paracrine stimulation) or in early cyst cells (autocrine stimulation) (Kiger et al. 2001; Tulina and Matunis 2001; Leatherman and DiNardo 2008). Upd activates the JAK-STAT cascade in CySCs to specify their self-renewal. Action of stat is required cell autonomously for CySC maintenance: Somatic CySCs made homogygous mutant for stat fail to maintain stem cell identity and instead commit to differentiation (Leatherman and DiNardo 2008). The transcriptional repressor zinc finger-homeodomain transcription factor $1(z f h-1)$ is likely to be a critical downstream target of the JAK-STAT pathway in CySCs. Zfh-1 protein accumulates in CySCs, and its levels drop precipitously as cyst cells codifferentiate with

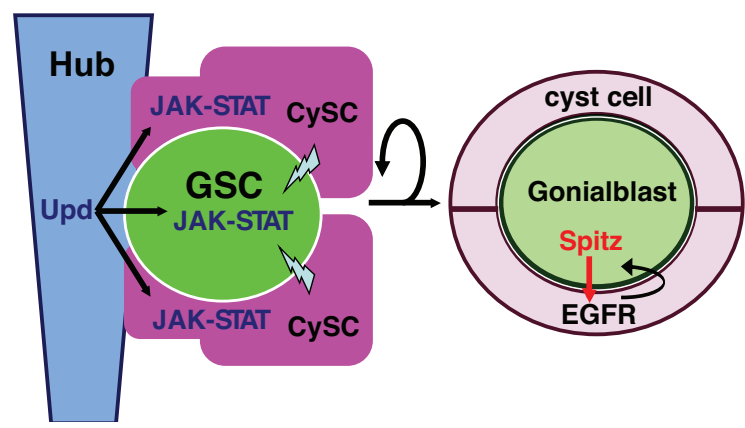

Figure 3. GSCs and CySCs require the Upd signal from the hub, and the hub and CySCs both contribute to the GSC niche. (Left) Diagram of the stem cell niche. Upd secreted by hub cells (blue) activates the JAK-STAT pathway, which is required cell autonomously in both the germ line (GSC) and cyst stem cells (CySC) for stem cell self-renewal. However, activation of STAT is not sufficient to instruct GSC self-renewal, which also requires as yet unknown signal(s) (lightning bolt) from the CySCs. (Right) Interactions between the progeny of somatic and germ line stem cells set up the functional unit of differentiation, the cyst. A Gb (light green), produced by asymmetric division of a GSC, signals via the epidermal growth factor receptor (EGFR) ligand Spitz to cyst cells (light pink) produced by asymmetric division of CySCs. Activation of EGFR triggers the somatic cyst cells to envelop the $\mathrm{Gb}$ and may also induce changes in gene expression in the cyst cells required for the germ cells to properly differentiate. 
spermatogonia. Zfh- 1 function is required cell autonomously for CySC maintenance. Importantly, ectopic expression of $Z f h-1$ in the soma is sufficient to promote CySC self-renewal and to block differentiation, even without concomitant up-regulation of JAK-STAT pathway activity in CySCs (Leatherman and DiNardo 2008).

\section{MULTIPLE CELL TYPES CONTRIBUTE TO NICHE FUNCTION}

Recent studies suggest that the niche that maintains male GSCs is complex, consisting of conversations among three different cell types: the GSCs, CySCs, and hub cells. Complex niches may be a feature of systems where multiple stem cell types must be maintained in close proximity, and their activities coordinated to produce progeny that form functional units and differentiate together. These mechanisms may be especially important in organs composed of cell types from different developmental origins.

The somatic CySCs contribute to the niche that maintains stem cell state in the germ line. Although activation of STAT in GSCs by Upd secreted from the hub is required for GSC self-renewal, it is not sufficient. Forced expression of a constitutively active allele of the JAK tyrosine kinase in early germ cells, leading to STAT activation, did not cause massive proliferation of GSCs at the expense of differentiation (Leatherman and DiNardo 2008; A.A. Kiger and M.T. Fuller, unpubl.). Strikingly, however, forced expression of constitutively active JAK in the cyst cell lineage resulted in overproliferation of GSCs or their immediate progeny and inhibited germ cell differentiation (Leatherman and DiNardo 2008). Similar results were seen when $Z f h-1$ was forcibly expressed in the somatic cyst cell lineage. Taken together, these data suggest that GSCs are maintained in the niche and instructed to self-renew by a combination of cytokine-like signals from the hub and as yet unknown $Z f h-1$-dependent signal(s) from the neighboring somatic CySCs (Fig. 3).

\section{STEM CELLS ORIENT TOWARD THE NICHE TO PROGRAM ASYMMETRIC DIVISION}

Drosophila male GSCs normally divide asymmetrically: The mitotic spindle is set up perpendicular to the hub-GSC interface so that, upon cytokinesis, one daughter remains in the niche and maintains stem cell identity, whereas the other daughter is displaced out of the niche and initiates differentiation (Fig. 2B,C). Thus, the mechanisms that polarize and orient the cytoskeleton in GSCs are key regulators of cell fate.

GSCs are physically attached to the hub by localized adherens junctions. Drosophila E-cadherin concentrates at the GSC cortex adjacent to the hub (Fig. 4A). $\beta$-catenin and APC2, a homolog of the adenomatous polyposis coli tumor suppressor, colocalize with E-cadherin at the adherens junctions, which provide a polarity cue toward which GSCs orient throughout the cell cycle (Yamashita et al. 2003). In $G_{1}$, the single centrosome in each GSC localizes near the cell cortex where the germ cell attaches to the hub (Fig. 4B, arrows). When the duplicated centro-
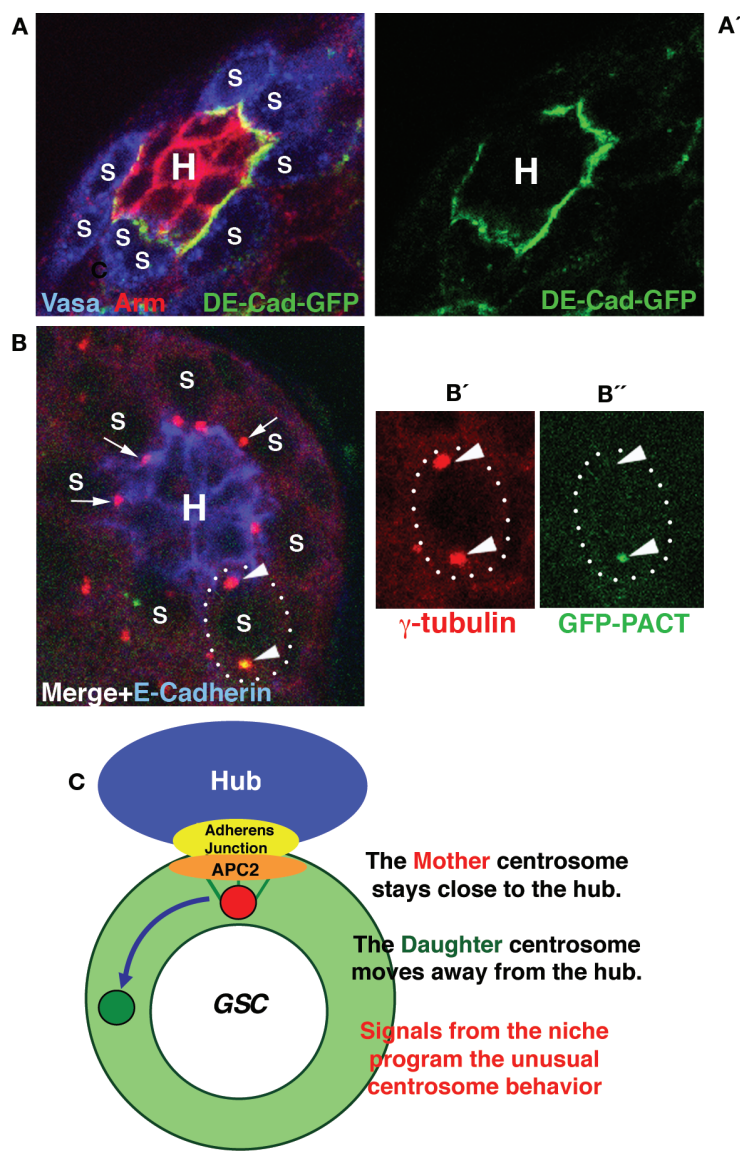

Figure 4. GSCs attach to and orient their centrosomes toward the hub. (A) Apical tip of a Drosophila testis expressing UAS-DEcadherin-GFP (green) exclusively in germ cells, under control of nos-GAL4. Note the localization of E-cadherin-GFP to the GSC cortex closest to the hub. GSCs (S) are clustered around the hub (germ cells are stained blue with anti-Vasa). Hub cells $(\mathrm{H})$ are stained with anti-Armadillo (red) but not anti-Vasa (blue). (B) End view of testis tip, showing hub cells $(\mathrm{H})$ outlined by anti-DE-cadherin staining (blue), surrounded by GSCs (S). (Arrows) Centrosomes in the interphase GSCs, visualized by staining with anti- $\gamma$-tubulin (red), lie next to the interface with the hub. (Dotted outline) Germ cell in $\mathrm{G}_{2}$ with two centrosomes (arrowheads), separated to opposite sides of the nucleus. The younger of the two centrosomes, pulse-labeled with GFP-PACT (visualized in $B$ and $B^{\prime \prime}$ ), has moved away from the hub, whereas the mother centrosome (not labeled with GFP-PACT) remains next to the hub. $(C)$ Model for how GSCs attach to and orient toward the hub. (Figure courtesy of Y. Yamashita.)

somes separate in $\mathrm{G}_{2}$, one stays next to the hub and the other migrates to the opposite side of the cell (Fig. 4B, arrowheads). The separation of centrosomes occurs unusually early in male GSCs: A substantial fraction of GSCs have centrosomes on opposite sides of the nucleus but have not yet built a spindle. APC2 function is required for GSCs to maintain proper orientation of the centrosomes toward the hub. The stereotyped position of the centrosomes in turn orients the mitotic spindle perpendicular to the GSC-hub cell interface, ensuring that the outcome of GSC divisions is asymmetric: One daughter inherits the attachment to the niche, remains subject to its signals, and renews stem cell identity, whereas the other 
is displaced away from the niche and initiates differentiation (Yamashita et al. 2003, 2007). Surprisingly, centrosomes are not oriented toward the niche throughout the cell cycle in female GSCs, indicating that spindle orientation is set up by a different mechanism in the germarium.

The two centrosomes in male GSCs have different characters and fates. Differential labeling of mother versus daughter centrosomes by transient expression of a green fluorescent protein (GFP)-tagged centriolar protein fragment during centrosome duplication revealed that the mother centrosome normally remains adjacent to the hub and is inherited by the GSC, whereas the daughter centrosome migrates to the opposite side of the cell and is inherited by the Gb (Fig. 4B). As a result, male GSCs maintain a centriolar Eve, a centriole that was assembled many cell generations earlier (Yamashita et al. 2007). The underlying cellular mechanism for the asymmetric inheritance of centrosomes during male GSC division may involve asymmetric maintenance of astral microtubule arrays. The mother centrosome appears to retain a robust astral microtubule array throughout the cell cycle, whereas the centrosome migrating to the opposite side of the cell had few associated astral microtubules until late in $G_{2}$, near the onset of mitosis. Consistent with this model, loss of attachment of astral microtubules to centrosomes in $\mathrm{cnn}$ mutant germ cells resulted in misoriented centrosomes and spindles (Yamashita et al. 2003) and randomized the positions of mother and daughter centrosomes (Yamashita et al. 2007).

The cellular mechanisms that maintain centrosome position and orient the mitotic spindle in stem cells are important because they ensure the asymmetric outcome of stem cell divisions within the niche. In the male germ line, Yamashita and colleagues hypothesize that a checkpoint mechanism blocks progression into mitosis unless a centrosome is properly situated next to the attachment to the hub (Cheng et al. 2008). Accumulation of GSCs that are arrested by this orientation checkpoint may contribute to the decrease in production of differentiating germ cells observed with aging.

\section{MANY INTERCELLULAR CONVERSATIONS REGULATE SELF-RENEWAL AND DIFFERENTIATION}

Two somatic cyst cells enclose each gonialblast to form a cyst (Fig. 3), the functional unit that initiates differentiation, starting with the onset of the spermatogonial transit-amplifying divisions. The cyst cells codifferentiate with the germ cells that they enclose (Gönczy et al. 1992). Bidirectional intercellular signaling between the germ cells and cyst cells is required to set up the cyst and to orchestrate codifferentiation of the two cell types.

Intercellular signaling via the epidermal growth factor receptor (EGFR) helps set up proper interactions between early germ cells and cyst cells. Early germ cells express the EGFR ligand Spitz that activates the EGFR in adjacent cyst cells (Schulz et al. 2002; Sarkar et al. 2007). Production of secreted Spitz by early germ cells requires the activity of stet, a germ-line-specific rhomboid class protease (Schulz et al. 2002; Sarkar et al. 2007). Activation of the EGFR along the Gb-cyst cell interface locally activates Vav, a guanine nucleotide exchange factor (GEF) for the small GTPase Rac1, which promotes local rearrangements in the cytoskeleton that induce cyst cells to send out processes and envelop the Gb (Sarkar et al. 2007). EGFR stimulation in cyst cells also activates a mitogen-activated protein kinase (MAPK)-dependent cascade that ultimately promotes differentiation of the enclosed germ cells (Kiger et al. 2000; Tran et al. 2000). One possibility is that enveloping cyst cells physically insulate early germ cells from selfrenewal signals from the hub or CySCs. Another possibility is that EGFR activation modulates expression in cyst cells of secreted factors that nonautonomously influence germ cell differentiation.

Signals from CySCs and early cyst cells may prevent premature differentiation of transit-amplifying germ cells by blocking expression of bag of marbles (bam), a key regulator of germ cell differentiation. In male germ cells, expression of bam normally initiates midway through the transit-amplification divisions, and wild-type function of bam is required for spermatogonia to cease mitotic divisions and initiate spermatocyte development (Gönczy et al. 1997). In the female germ line, bam transcription is silenced in GSCs by TGF- $\beta$ signaling from the niche (Xie and Spradling 2000; Spradling et al., this volume), which causes an activated Smad-CoSmad complex containing Mad and Medea to bind cis-acting control elements just downstream from the bam transcription start site, blocking bam expression in female GSCs (Chen and McKearin 2003a,b). Expression of bam commences as female germ cells exit the niche and is both necessary and sufficient for the cells to initiate transit-amplifying divisions and differentiation (McKearin and Ohlstein 1995). In contrast, bam function is not required for male GSCs to divide asymmetrically or for spermatogonia to undergo transit-amplifying divisions (Gönczy et al. 1997). However, bam expression must be repressed in GSCs and early germ cells to allow survival and prevent premature differentiation. Forced high-level expression of bam in early male germ cells caused arrest and cell death (Schulz et al. 2004).

In males, TGF- $\beta$ signaling from soma to germ line appears to inhibit precocious expression of bam in GSCs and spermatogonial cysts. Intracellular components of the TGF- $\beta$ signal transduction machinery, including the receptors punt and thick veins, are required cell autonomously for male GSC maintenance (Shivdasani and Ingham 2003; Bunt and Hime 2004; Kawase et al. 2004). Hub and early cyst cells express the TGF- $\beta$ class signaling ligand glass bottom boat ( $\mathrm{gbb}$ ), which is required for GSC survival (Shivdasani and Ingham 2003). A likely scenario is that the hub, CySCs, and early cyst cells secrete Gbb and potentially other TGF- $\beta$ ligands, activating the TGF- $\beta$ signal transduction pathway in neighboring germ cells and causing transcriptional repression of bam. The resulting silencing of bam in early germ cells allows GSC and Gb survival. The mechanisms that downregulate $g b b$ expression as cyst cells differentiate to allow expression of bam in late spermatogonia are not known, but it is tempting to speculate that a germ line to soma signal, perhaps germ-line-derived Spitz activating EGFR in cyst cells, might be involved. 


\section{TRANSIT-AMPLIFYING CELLS CAN REVERT TO STEM CELL IDENTITY}

Remarkably, male and female germ cells undergoing transit-amplifying divisions can and indeed often do reoccupy the niche and revert back to stem cell identity (Brawley and Matunis 2004; Kai and Spradling 2004; Cheng et al. 2008). Reversion of transit-amplifying cells to stem cell state may provide a mechanism to replace stem cells lost from the niche by normal turnover or damage and may be especially important in cases where stereotyped spindle orientation normally prevents a symmetric outcome of stem cell divisions in the niche.

Although transit-amplifying cells are clearly morphologically and behaviorally different from GSCs, they are not yet irreversibly committed to differentiation. Dedifferentiation of spermatogonia requires STAT activity. Adult males conditionally mutant for stat maintained stem cells at permissive temperature, but they rapidly lost both GSCs and CySCs to differentiation when shifted to nonpermissive temperature. However, if the flies were returned to permissive conditions within a few days, transit-amplifying spermatogonial cysts adjacent to the hub broke apart and provided germ cells that regained stem cell behavior, reconstituting the vacated niche (Brawley and Matunis 2004). Unlike spermatogonia, spermatocytes did not appear to be able to revert to stem cell identity.

Genetic marking experiments demonstrated that transitamplifying germ cells that have progressed far enough to turn on bam expression can repopulate the niche and revert to stem cell behavior, even without drastic clearing of the niche and manipulations of niche signaling pathways (Cheng et al. 2008). The frequency of stem cell replacement by transit-amplifying cell reversion increased when flies were irradiated, suggesting that dedifferentiation of transit-amplifying cells may be an important mechanism for restoring stem cells to the niche after damage. Interestingly, dedifferentiated GSCs exhibit misoriented centrosomes and undergo cell cycle arrest until proper centrosome orientation toward the hub is reestablished (Cheng et al. 2008). Thus, it may take some time under the influence of niche signals for returning germ cells to express the molecules responsible for the unusual cytoskeletal behavior that anchors the oldest centrosome near the junction between the GSC and the hub.

\section{THE SWITCH TO TERMINAL DIFFERENTIATION REPROGRAMS THE TRANSCRIPTION MACHINERY}

Once the germ cells have completed four rounds of transit-amplifying divisions, the resulting 16 cells exit the mitotic division program and initiate spermatocyte growth and differentiation. This cell state change involves dramatic remodeling of the transcriptional landscape: Many genes expressed in mitotic precursor cells are turned off and many new genes are expressed, some for the first time in the development of the animal. Quite frequently, genes normally thought to serve a general or housekeeping function have homologs in the genome that are expressed in spermatocytes but not in the proliferating precursor cells. These include homologs encoding sub- units of the proteosome (Yuan et al. 1996), mitochondrial import complex (Hwa et al. 2004), and translation initiation machinery (Baker and Fuller 2007). The emerging picture is that the switch to the spermatocyte state initiates a multistep transcriptional hierarchy that controls spermatocyte growth and development, the meiotic cell cycle, and eventually the expression of hundreds of genes required for spermatid differentiation.

A key question in stem cell lineages is how terminal differentiation genes kept silent in precursor cells are activated in the correct cell type and at the right time during differentiation. Studies on the meiotic arrest genes in Drosophila have provided insight into the underlying regulatory mechanisms at work in the male germ line. In meiotic-arrest mutant males, GSCs, transit-amplifying cells, and spermatocytes are formed. Although they grow to normal size and have normal morphology, the mutant spermatocytes arrest at the $\mathrm{G}_{2} / \mathrm{M}$ transition of meiosis I and fail to initiate spermatid differentiation. The phenotype of the Drosophila meiotic-arrest mutants is strikingly similar to the clinical description of meiosis I maturation arrest azoospermia, a common form of human idiopathic male infertility (Meyer et al. 1992). Wild-type function of the meiotic arrest genes is required for normal expression of hundreds of genes involved in postmeiotic spermatid differentiation. The defect in differentiation is independent of cell cycle arrest, because spermatid differentiation genes that are direct targets of the meiotic-arrest genes are normally transcribed starting at the onset of the primary spermatocyte period, days before the $\mathrm{G}_{2} / \mathrm{M}$ transition of meiosis I.

Phenotypic analysis and molecular cloning revealed that the Drosophila meiotic-arrest genes fall into two functional classes, encoding components of two separate regulatory complexes. The first, represented by aly, encodes components of tMAC, a testis-specific variant of MIP/dREAM (White-Cooper et al. 2000; Perezgasga et al. 2004; Beall et al. 2007; Jiang et al. 2007), a multisubunit complex conserved from worms (the SynMuv pathway) to mammals (E2F/Rb/Myb interacting proteins). MIP/dREAM complexes have an important but not yet understood role in cell cycle control and expression of developmentally controlled genes (Korenjak et al. 2004). Strikingly, the aly class meiotic-arrest genes encode spermatocyte-specific homologs of generally expressed MIP/dREAM subunits, presumably endowing the tMAC complex with unique properties not shared by the MIP/dREAM transcriptional regulator. tMAC function is required for transcription in primary spermatocytes of meiotic cell cycle control genes such as cyclin B, boule, and the CDC25 cell cycle phosphatase twine, as well as spermatid differentiation genes (WhiteCooper et al. 1998).

The second class of meiotic arrest genes, represented by can, $n h t$, rye, $s a$, and mia, all encode spermatocytespecific TBP-associated factor (TAF) homologs (Hiller et al. 2001, 2004). TAFs interact with the TATA-box binding protein (TBP) to form the general transcription factor TFIID that interprets and integrates molecular signals regulating the core RNA polymerase II (Pol II) machinery for transcriptional initiation (for review, see Hochheimer and Tjian 2003). Certain TAFs are also core components of histone acetylase complexes such as SAGA (Spt-Ada- 
Gcn5-acetyltransferase) (Struhl et al. 1998) or bind stoichiometrically to the Polycomb transcriptional silencing complex in some tissues (Saurin et al. 2001). Wild-type function of the testis TAFs (tTAFs) is required for robust transcription in spermatocytes of many of the same spermatid differentiation genes that require tMAC action (White-Cooper et al. 1998; Hiller et al. 2004). Strikingly, regulation of transcription by the tTAFs and tMAC is gene selective: Although transcription of spermatid differentiation genes is severely affected in mutant spermatocytes, many other genes are expressed normally in the same cells. Thus, tissue-specific homologs of general Pol II transcription machinery components regulate geneselective, cell-type-specific transcriptional programs for terminal differentiation in the Drosophila male germ cell lineage (White-Cooper et al. 1998; Hiller et al. 2001).

Emerging evidence indicates that tissue-specific TAFs and other core promotor recognition machinery components control cell-type-specific gene expression programs in a variety of developmental contexts. Incorporation of tissue-specific TAFs may alter the properties of TFIID, enabling the modified complex to direct widely expressed transcription factors to activate specific target genes in certain tissues. In mice, TAF4b, a tissue-specific alternate form of TAF4, is required for maintenance of spermatogenesis (Falender et al. 2005) and differentiation of mouse ovarian granulosa cells (Freiman et al. 2001). Substitution of TAF4b for one of the two TAF4 subunits in TFIID strongly facilitates transcriptional activation by c-Jun at selected promoters (Liu et al. 2008), activating tissue-specific gene-selective transcription programs in cells where both c-Jun and TAF4b are expressed. In a more dramatic core machinery switch, during muscle development, TFIID is down-regulated and instead TAF3 and the TBP homolog TRF3 turn on transcription of myogenin and other genes required for differentiation of myoblasts into myotubes (Deato and Tjian 2007 and this volume).

In the Drosophila male GSC lineage, expression of terminal differentiation genes in spermatocytes depends on cell-type-specific modification of core promotor recognition machinery components and chromatin regulatory complexes. These in turn selectively promote expression of genes required for meiosis and spermiogenesis in germ cells committed to terminal differentiation. The cell-type- and stage-specific expression of tTAFs and tMAC components in spermatocytes begs the question of how expression of these factors is regulated by the stem cell lineage program. We imagine a transcriptional cascade where regulatory factor(s) expressed as part of the switch to spermatocyte state induce expression of the tTAFs and spermatocyte-specific tMAC components. These in turn act together to allow transcription of terminal differentiation genes.

\section{TERMINAL DIFFERENTIATION INVOLVES CELL-TYPE-SPECIFIC MECHANISMS TO COUNTERACT REPRESSION BY POLYCOMB}

The testis TAFs and tMAC may facilitate expression of terminal differentiation genes by reversing repression by Polycomb epigenetic transcriptional silencing machinery. Chromatin immunoprecipitation (ChIP) experiments on
tTAF mutant testes showed Polycomb protein at the promoter regions of all three of the terminal differentiation genes assayed. In wild-type testes, these promoters were instead occupied by the tTAFs, and the differentiation genes were expressed (Chen et al. 2005). The tTAFs also appear to control subnuclear localization of the Polycomb complex PRC1. In wild-type spermatocytes, tTAF proteins accumulate in a subdomain of the nucleolus. PRC1 components localized to the same nucleolar subcompartment in a tTAF-dependent manner (Chen et al. 2005). Taken together, these results suggest that the tTAFs might bind PRC1 and sequester the complex to the nucleolus. Sequestering PRC1 may promote expression of spermatid differentiation genes by preventing rebinding of free PRC1 to target gene promoters. Alternatively, PRC1 may have a novel function in the nucleolus.

Recent studies in mammalian embryonic stem cells suggest that many genes required for lineage commitment and tissue-specific differentiation programs are silenced in embryonic stem cells by Polycomb group (PcG) machinery. Loss of PcG function in ES cells causes inappropriate expression of developmental regulators and defects in differentiation of particular cell types (Boyer et al. 2006; Lee et al. 2006; Chamberlain et al. 2008). PcG machinery appears to have critical roles in decisions between proliferating precursor cell fate and terminal differentiation in mammalian adult stem cell lineages as well. In mice, loss of function of the PcG protein Bmi-1 causes loss of long-term self-renewing capacity in hematopoietic and neural stem cells. Additionally, Bmil expression is up-regulated in certain leukemias (Lessard and Sauvageau 2003; Molofsky et al. 2003; Park et al. 2003). If PcG activity blocks expression of differentiation genes in precursor cells, a major question is how this epigenetically silenced state is normally reversed to allow expression of differentiation genes appropriate to distinct cell types and developmental stages. Studies on the role of the testis TAFs and tMAC in the Drosophila male GSC lineage provide a model for how cell-type-specific forms of core transcriptional regulatory machinery might have a role in reversing epigenetic silencing to activate cell-typespecific transcription programs as precursor cells initiate and execute terminal differentiation.

\section{DISCUSSION}

Studies of model stem cell lineages in vivo are yielding important lessons for understanding tissue homeostasis, aging, regenerative medicine, and cancer. The importance of the niche in controlling stem cell behavior is paramount. However, we must understand the complexities of natural stem cell niches before we can hope to mimic them for expansion of adult stem cells in vitro, for construction of artificial niches for therapeutic applications, or even for understanding the relationship between stem cell lineages and cancer. Indeed, recent work on basal cell carcinoma indicates that tumor cells are maintained by close-range signals from accompanying stromal cells, which provide an ersatz niche (Sneddon et al. 2006). The picture emerging from work on GSC niches in Drosophila points toward a self-sustaining and mutually reinforcing community of 
several cell types engaged in close-range signaling conversations that influence the fate choices of neighbors within the niche (see also Spradling et al., this volume).

A second important lesson is the ability of cells to embark on transit-amplifying divisions but still be able to revert to the stem cell state, given access to an empty niche. Replacement of stem cells by dedifferentiation of transitamplifying cells occurs in the female (Kai and Spradling 2004) as well as the male germ line in Drosophila and also appears to occur during mammalian spermatogenesis (Nakagawa et al. 2007; also see Yoshida, this volume). This ability means that transplantation assays may detect not only the most fundamental stem cells, but also possibly early transit-amplifying cells that have initiated but are not yet committed to differentiation. It will be interesting to find out if this underlies the recent discovery of two interconverting populations of hematopoietic stem cells, one deeply quiescent and one more rapidly proliferating (Wilson et al. 2008).

As we understand more clearly the signaling pathways and regulatory strategies that maintain the stem cell state in adult tissues, it will be interesting to compare these to the signals that maintain pluripotency of embryonic stem cells. In the case of the Drosophila male germ line, stem cell selfrenewal and maintenance requires activation of STAT by a cytokine-like signaling ligand as well as repression of differentiation by TGF- $\beta$ class signals from neighboring cells. Without the TGF- $\beta$ signal, GSCs precociously express the lineage-specific differentiation factor bam. The cooperative action of JAK-STAT and TGF- $\beta$ pathways in male GSC self-renewal is reminiscent of the dependence of murine embryonic stem cells on LIF/STAT3 and BMP/ Smad signaling where, again, these pathways sustain the stem cell state by inhibiting cells from adopting lineagespecific fates (Ying et al. 2003). A second intriguing parallel between adult and embryonic stem cell lineages may lie in a need for mechanisms to reverse Polycomb-mediated repression by allowing context-appropriate expression of terminal differentiation genes.

\section{ACKNOWLEGMENTS}

We thank many present and former members of the Fuller lab, including Alexis Bailey, Catherine Baker, Xin Chen, Edith Glusman, Megan Insco, D. Leanne Jones, Amy Kiger, Chenggang Lu, Anthony Mahowald, Jose Morillo Prado, Alicia Shields, Allyson Campbell Spence, Vidhya Srinivasan, and Yukiko Yamashita, whose scientific discussions and work contributed greatly to the development of these ideas. E.D. was supported by the Smith Stanford Graduate Fellowship and predoctoral fellowships from the National Science Foundation and the American Heart Association Western Affiliate. M.T.F. is the ReedHodgson Professor of Human Biology at Stanford. This work was supported by National Institutes of Health grants 1 R01 GM080501 and 3 RO1 GM061986 to M.T.F.

\section{REFERENCES}

Baker, C.C. and Fuller, M.T. 2007. Translational control of meiotic cell cycle progression and spermatid differentiation in male germ cells by a novel eIF4G homolog. Development
134: 2863-2869.

Beall, E.L., Lewis, P.W., Bell, M., Rocha, M., Jones, D.L., and Botchan, M.R. 2007. Discovery of tMAC: A Drosophila testis-specific meiotic arrest complex paralogous to MybMuv B. Genes Dev. 21: 904-919.

Boyer, L.A., Plath, K., Zeitlinger, J., Brambrink, T., Medeiros, L.A., Lee, T.I., Levine, S.S., Wernig, M., Tajonar, A., Ray, M.K., et al. 2006. Polycomb complexes repress developmental regulators in murine embryonic stem cells. Nature 441: 349-353.

Boyle, M., Wong, C., Rocha, M., and Jones, D.L. 2007. Decline in self-renewal factors contributes to aging of the stem cell niche in the Drosophila testis. Cell Stem Cell 1: 470-478.

Brawley, C. and Matunis, E. 2004. Regeneration of male germline stem cells by spermatogonial dedifferentiation in vivo. Science 304: 1331-1334.

Bunt, S.M. and Hime, G.R. 2004. Ectopic activation of Dpp signalling in the male Drosophila germline inhibits germ cell differentiation. Genesis 39: 84-93.

Chamberlain, S.J., Yee, D., and Magnuson, T. 2008. Polycomb repressive complex 2 is dispensable for maintenance of embryonic stem cell pluripotency. Stem Cells 26: 1496-1505.

Chen, D. and McKearin, D. 2003a. Dpp signaling silences bam transcription directly to establish asymmetric divisions of germline stem cells. Curr. Biol. 13: 1786-1791.

Chen, D. and McKearin, D.M. 2003b. A discrete transcriptional silencer in the bam gene determines asymmetric division of the Drosophila germline stem cell. Development 130: 1159-1170.

Chen, X., Hiller, M., Sancak, Y., and Fuller, M.T. 2005. Tissuespecific TAFs counteract Polycomb to turn on terminal differentiation. Science 310: 869-872.

Cheng, J., Turkel, N., Hemati, N., Fuller, M.T., Hunt, A.J., and Yamashita, Y.M. 2008. Centrosome misorientation reduces stem cell division during ageing. Nature 456: 599-604.

Clarke, M.F. and Fuller, M. 2006. Stem cells and cancer: Two faces of Eve. Cell 124: 1111-1115.

Deato, M.D. and Tjian, R. 2007. Switching of the core transcription machinery during myogenesis. Genes Dev. 21: 2137-2149.

Falender, A.E., Freiman, R.N., Geles, K.G., Lo, K.C., Hwang, K., Lamb, D.J., Morris, P.L., Tjian, R., and Richards, J.S. 2005. Maintenance of spermatogenesis requires TAF $4 b$, a gonad-specific subunit of TFIID. Genes Dev. 19: 794-803.

Freiman, R.N., Albright, S.R., Zheng, S., Sha, W.C., Hammer, R.E., and Tjian, R. 2001. Requirement of tissue-selective TBP-associated factor $\mathrm{TAF}_{\mathrm{II}} 105$ in ovarian development. Science 293: 2084-2087.

Fuller, M.T. 1993. Spermatogenesis. In The development of Drosophila melanogaster (ed. M. Bate and A. MartinezArias), pp. 71-147. New York, Cold Spring Harbor Laboratory Press, Cold Spring Harbor, New York.

Fuller, M.T. and Spradling, A.C. 2007. Male and female Drosophila germline stem cells: Two versions of immortality. Science 316: 402-404.

Gönczy, P. and DiNardo, S. 1996. The germ line regulates somatic cyst cell proliferation and fate during Drosophila spermatogenesis. Development 122: 2437-2447.

Gönczy, P., Matunis, E., and DiNardo, S. 1997. bag-of-marbles and benign gonial cell neoplasm act in the germline to restrict proliferation during Drosophila spermatogenesis. Development 124: 4361-4371.

Gönczy, P., Viswanathan, S., and DiNardo, S. 1992. Probing spermatogenesis in Drosophila with P-element enhancer detectors. Development 114: 89-98.

Hecht, N.B. 1993. Gene expression during male germ cell development. In Cell and molecular biology of the testis (ed. C. Desjardins and L.L. Ewing), pp. 400-432. Oxford University Press, New York.

Hiller, M., Chen, X., Pringle, M.J., Suchorolski, M., Sancak, Y., Viswanathan, S., Bolival, B., Lin, T.Y., Marino, S., and Fuller, M.T. 2004. Testis-specific TAF homologs collaborate to control a tissue-specific transcription program. Development 131: 5297-5308.

Hiller, M.A., Lin, T.Y., Wood, C., and Fuller, M.T. 2001. Developmental regulation of transcription by a tissue-specific TAF homolog. Genes Dev. 15: 1021-1030. 
Hochheimer, A. and Tjian, R. 2003. Diversified transcription initiation complexes expand promoter selectivity and tissuespecific gene expression. Genes Dev. 17: 1309-1320.

Hwa, J.J., Zhu, A.J., Hiller, M.A., Kon, C.Y., Fuller, M.T., and Santel, A. 2004. Germ-line specific variants of components of the mitochondrial outer membrane import machinery in Drosophila. FEBS Lett. 572: 141-146.

Jiang, J., Benson, E., Bausek, N., Doggett, K., and WhiteCooper, H. 2007. Tombola, a tesmin/TSO1-family protein, regulates transcriptional activation in the Drosophila male germline and physically interacts with Always early. Development 134: 1549-1559.

Kai, T. and Spradling, A. 2004. Differentiating germ cells can revert into functional stem cells in Drosophila melanogaster ovaries. Nature 428: 564-569.

Kawase, E., Wong, M.D., Ding, B.C., and Xie, X. 2004. Gbb/Bmp signaling is essential for maintaining germline stem cells and for repressing bam transcription in the Drosophila testis. Development 131: 1365-1375.

Kiger, A.A., White-Cooper, H., and Fuller, M.T. 2000. Somatic support cells restrict germline stem cell self-renewal and promote differentiation. Nature 407: 750-754.

Kiger, A.A., Jones, D.L., Schulz, C., Rogers, M.B., and Fuller, M.T. 2001. Stem cell self-renewal specified by JAK-STAT activation in response to a support cell cue. Science 294: 2542-2545.

Korenjak, M., Taylor-Harding, B., Binne, U.K., Satterlee, J.S., Stevaux, O., Aasland, R., White-Cooper, H., Dyson, N., and Brehm, A. 2004. Native E2F/RBF complexes contain Mybinteracting proteins and repress transcription of developmentally controlled E2F target genes. Cell 119: 181-193.

Leatherman, J.L. and DiNardo, S. 2008. Zfh-1 controls somatic stem cell self-renewal in the Drosophila testis and nonautonomously influences germline stem cell self-renewal. Cell Stem Cell 3: 44-54.

Le Bras, S. and Van Doren, M. 2006. Development of the male germline stem cell niche in Drosophila. Dev. Biol. 294: 92-103.

Lee, T.I., Jenner, R.G., Boyer, L.A., Guenther, M.G., Levine, S.S., Kumar, R.M., Chevalier, B., Johnstone, S.E., Cole, M.F., Isono, K., et al. 2006. Control of developmental regulators by Polycomb in human embryonic stem cells. Cell 125: 301-313.

Lessard, J. and Sauvageau, G. 2003. Bmi-1 determines the proliferative capacity of normal and leukaemic stem cells. Nature 423: $255-260$.

Liu, W.L., Coleman, R.A., Grob, P., King, D.S., Florens, L., Washburn, M.P., Geles, K.G., Yang, J.L., Ramey, V., Nogales, E., and Tjian, R. 2008. Structural changes in TAF4b-TFIID correlate with promoter selectivity. Mol. Cell 29: 81-91.

McKearin, D. and Ohlstein, B. 1995. A role for the Drosophila Bag-of-marbles protein in the differentiation of cystoblasts from germline stem cells. Development 121: 2937-2947.

Meyer, J.M., Maetz, J.L., and Rumpler, Y. 1992. Cellular relationship impairment in maturation arrest of human spermatogenesis: An ultrastructural study. Histopathology 21: 25-33.

Molofsky, A.V., Pardal. R., Iwashita, T., Park, I.K., Clarke, M.F., and Morrison, S.J. 2003. Bmi-1 dependence distinguishes neural stem cell self-renewal from progenitor proliferation. Nature 425: 962-967.

Nakagawa, T., Nabeshima, Y., and Yoshida, S. 2007. Functional identification of the actual and potential stem cell compartments in mouse spermatogenesis. Dev. Cell 12: 195-206.

Park, I.K., Qian, D., Kiel, M., Becker, M.W., Pihalja, M., Weissman, I.L., Morrison, S.J., and Clarke, M.F. 2003. Bmi-1 is required for maintenance of adult self-renewing haematopoietic stem cells. Nature 423: 302-305.

Perezgasga, L., Jiang, J., Bolival, Jr., B., Hiller, M., Benson, E., Fuller, M.T., and White-Cooper, H. 2004. Regulation of transcription of meiotic cell cycle and terminal differentiation genes by the testis-specific Zn-finger protein matotopetli. Development 131: 1691-1702.

Sarkar, A., Parikh, N., Hearn, S.A., Fuller, M.T., Tazuke, S.I., and Schulz, C. 2007. Antagonistic roles of Rac and Rho in organizing the germ cell microenvironment. Curr. Biol. 17: $1253-1258$.

Saurin, A.J., Shao, Z., Erdjument-Bromage, H., Tempst, P., and
Kingston, R.E. 2001. A Drosophila Polycomb group complex includes Zeste and dTAFII proteins. Nature 412: 655-660.

Schulz, C., Wood, C.G., Jones, D.L., Tazuke, S.I., and Fuller, M.T. 2002. Signaling from germ cells mediated by the rhomboid homologue stet organizes encapsulation by somatic support cells. Development 129: 4523-4534.

Schulz, C., Kiger, A.A., Tazuke, S.I., Yamashita, Y.M., PantalenaFilho, L.C., Wood, C.G., Jones, D.L., and Fuller, M.T. 2004 A misexpression screen reveals effects of bag-of-marbles and TGF $\beta$ class signaling on the Drosophila male germ line stem cell lineage. Genetics 167: 707-723.

Shivdasani, A.A. and Ingham, P.W. 2003. Regulation of stem cell maintenance and transit amplifying cell proliferation by TGF- $\beta$ signaling in Drosophila spermatogenesis. Curr. Biol. 13: 2065-2072.

Sneddon, J.B., Zhen, H.H., Montgomery, K., van de Rijn, M., Tward, A.D., West, R., Gladstone, H., Chang, H.Y., Morganroth, G.S., Oro, A.E., and Brown, P.O. 2006. Bone morphogenetic protein antagonist gremlin 1 is widely expressed by cancer-associated stromal cells and can promote tumor cell proliferation. Proc. Natl. Acad. Sci. 103: 14842-14847.

Spradling, A., Drummond-Barbosa, D., and Kai, T. 2001. Stem cells find their niche. Nature 414: 98-104.

Struhl, K., Kadosh, D., Keaveney, M., Kuras, L., and Moqtaderi, Z. 1998. Activation and repression mechanisms in yeast. Cold Spring Harbor Symp. Quant. Biol. 63: 413-421.

Tran, J., Brenner, T.J., and DiNardo, S. 2000. Somatic control over the germline stem cell lineage during Drosophila spermatogenesis. Nature 407: 754-757.

Tulina, N. and Matunis, E. 2001. Control of stem cell selfrenewal in Drosophila spermatogenesis by JAK-STAT signaling. Science 294: 2546-2549.

Voog, J., D'Alterio, C., and Jones, D.L. 2008 Multipotent somatic stem cells contribute to the stem cell niche in the Drosophila testis. Nature 454: 1132-1136.

Watt, F.M. and Hogan, B.L. 2000. Out of Eden: Stem cells and their niches. Science 287: 1427-1430.

White-Cooper, H., Leroy, D., MacQueen, A., and Fuller, M.T. 2000. Transcription of meiotic cell cycle and terminal differentiation genes depends on a conserved chromatin associated protein, whose nuclear localisation is regulated. Development 127: 5463-5473.

White-Cooper, H., Schafer, M.A., Alphey, L.S., and Fuller, M.T. 1998. Transcriptional and post-transcriptional control mechanisms coordinate the onset of spermatid differentiation with meiosis I in Drosophila. Development 125: 125-134.

Wilson, A., Laurenti, E., Oser, G., van der Wath, R.C., BlancoBose, W., Jaworski, M., Offner, S., Dunant, C.F., Eshkind, L., Bockamp, E., et al. 2008. Hematopoietic stem cells reversibly switch from dormancy to self-renewal during homeostasis and repair. Cell 135: 1118-1129.

Xie, T. and Spradling, A.C. 1998. decapentaplegic is essential for the maintenance and division of germline stem cells in the Drosophila ovary. Cell 94: 251-260.

Xie, T. and Spradling, A.C. 2000. A niche maintaining germ line stem cells in the Drosophila ovary. Science 290: 328-330.

Yamashita, Y.M. and Fuller, M.T. 2005. Asymmetric stem cell division and function of the niche in the Drosophila male germ line. Int. J. Hematol. 82: 377-380.

Yamashita, Y.M., Fuller, M.T., and Jones, D.L. 2005. Signaling in stem cell niches: Lessons from the Drosophila germline. $J$. Cell Sci. 118: 665-672.

Yamashita, Y.M., Jones, D.L., and Fuller, M.T. 2003. Orientation of asymmetric stem cell division by the APC tumor suppressor and centrosome. Science 301: 1547-1550.

Yamashita, Y.M., Mahowald, A.P., Perlin, J.R., and Fuller, M.T. 2007. Asymmetric inheritance of mother versus daughter centrosome in stem cell division. Science 315: 518-521.

Ying, Q.L., Nichols, J., Chambers, I., and Smith, A. 2003. BMP induction of Id proteins suppresses differentiation and sustains embryonic stem cell self-renewal in collaboration with STAT3. Cell 115: 281-292.

Yuan, X., Miller, M. and Belote, J.M. 1996 Duplicated proteasome subunit genes in Drosophila melanogaster encoding testes-specific isoforms. Genetics 144: 147-157. 


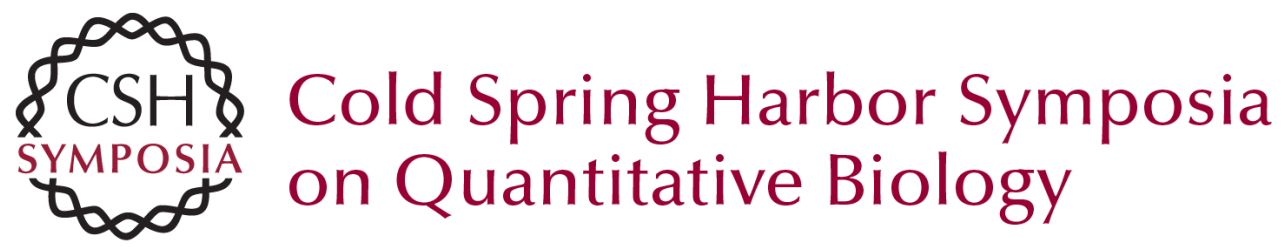

\title{
Regulation of Self-renewal and Differentiation in Adult Stem Cell Lineages: Lessons from the Drosophila Male Germ Line
}

\author{
E.L. Davies and M.T. Fuller
}

Cold Spring Harb Symp Quant Biol 2008 73: 137-145 originally published online March 27, 2009 Access the most recent version at doi:10.1101/sqb.2008.73.063

References This article cites 63 articles, 33 of which can be accessed free at: http://symposium.cshlp.org/content/73/137.full.html\#ref-list-1

\section{License}

Email Alerting

Receive free email alerts when new articles cite this article - sign up in the box at the Service top right corner of the article or click here.

To subscribe to Cold Spring Harbor Symposia on Quantitative Biology go to: http://symposium.cshlp.org/subscriptions 